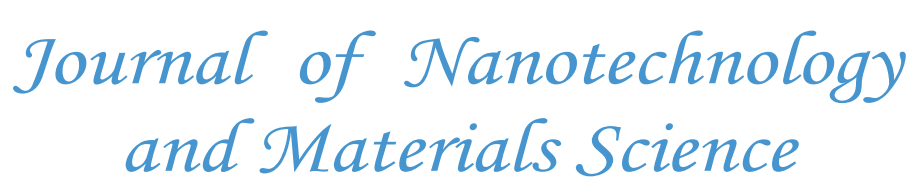

\title{
Effect of Lyophilization on the Size and Polydispersity of Unilamellar and Multilamellar Liposomes
}

\author{
Mahshid Shokri', Mohmood Tavallaie ${ }^{2 *}$, Seyed Mostafa Hosseini \\ ${ }^{1}$ Department of Biomedical Engineering, Science and Research Branch, Islamic Azad University, Tehran, Iran \\ ${ }^{2}$ Human Genetic Research Centre, Baqiyatallah Medical Sciences University, Tehran, Iran
}

*Corresponding author: Mohmood Tavallaie, Human Genetic Research Centre, Baqiyatallah Medical Sciences University, Tehran, Iran, Tel: (+ 98) 26403292; Fax: (+ 98) 26413382; E-mail: tavalla.mah@gmail.com

\begin{abstract}
With the emergence of drug delivery systems, liposome's attracted many attentions as drug and gene delivery vehicles. In this regard, long-term stability of liposomes becomes important in the clinical use of these particles and their success in the market of therapeutics. Lyophilisation is one of the most common methods for preservation of liposome nanoparticles. This process may change some key properties of particles such as size and polydispersity. Hence, in this article we investigated the effects of lyophilization process on these two parameters. A multilamellar liposomal formulation of DOTAP/DOPE/Cholesterol was fabricated through modified lipid film hydration method while extrusion was employed to obtain unilamellar ones. Lyophilization was performed in the presence of sucrose used as the cryoprotectant. The results show that after lyophilization, the mean particle size of both multilamellar and unilamellar vesicles had no significant change $(\mathrm{p}>0.1)$ in contrast to poly dispersity index $(\mathrm{p}<0.05)$
\end{abstract}

Received date: September 27, 2016

Accepted date: October 24, 2016

Published date: October 31, 2016

Citation: Tavallaie, M., et al. Effect of Lyophilization on the Size and Polydispersity of Unilamellar and Multilamellar Liposomes. (2016) J Nanotech Mater Sci 3(2): $37-40$.

DOI: $10.15436 / 2377-1372.16 .1139$

Keywords: Liposome; Lyophilization; Delivery vehicle; Unilamellar vesicle; Multilamellar vesicle

\section{Introduction}

Long-term stability of colloidal polymeric nanoparticles loaded with different drugs has been always a significant factor determining clinical success of this therapeutics. Lyophilization is regarded as one of the most important methods of preservation of nanoparticles. Hence, many efforts have been made to determine significant factors in the process of lyophilization and their impact on the final stability has been discussed ${ }^{[1-3]}$.

One of the main classes of colloidal nano and micro particles used for the purpose of delivering therapeutic agents to the tissues are liposomes. Loading of different hydrophobic and hydrophilic drugs into liposomes with high encapsulation efficiency is possible due to the multilayered structure of this type of carriers $^{[4,5]}$.

Many developments have been made in the field of liposomal drug delivery. A number of these formulations ap- proved clinically and entered the market successfully. Therefore, a significant subject is the choice of a suitable method to stabilize these particles to provide a long term shelf life of these kinds of products. Lyophilization has been used repeatedly as an alternative method of preserving nanoparticle suspension. Although this method has been showed to be suitable for long-term stability of liposomes, lyophilization can alter the organization of liposome bilayer or the size of particles ${ }^{[6-10]}$. It is obvious that particle size impact on the drug release from nano carriers to a great extent. Hence, it is very important to compare the size of liposomes before and after freeze drying process.

In this article, a liposomal formulation of DOTAP/ DOPE/Cholesterol was fabricated. Among different methods of liposome preparation including hydration of a lipid film ${ }^{[11]}$, dehydration-rehydration $(\mathrm{DRV})^{[12]}$, ethanolic injection ${ }^{[13]}$, Re- 
verse-Phase Evaporation $(\mathrm{REV})^{[14]}$, or the detergent dialysis technique $^{[15]}$. Modified lipid film hydration ${ }^{[16]}$ was selected and followed by membrane extrusion to obtain unilamellar liposomes $^{[17]}$. The formulations were freeze-dried in the presence of sucrose as the cryoprotectant and the size and polydispersity of particles were compared before and after lyophilization.

\section{Materials and Methods}

Materials: 1, 2-di-O-octadecenyl-3-trimethylammonium propane (DOTAP) and 1,2-dioleoyl-sn-glycero-3-phosphoethanolamine (DOPE) were purchased from Avanti Polar lipids. Cholesterol and Sucrose were obtained from Sigma-Aldrich and Alfa Aesar, respectively. All the solvents were of analytical grade.

Fabrication of liposomes: Cationic liposomes were fabricated using a modified lipid film hydration method. Briefly, DOTAP/ DOPE/cholesterol with a molar ratio of 1:1:2 were dissolved in chloroform (total concentration of $5 \mathrm{mg} / \mathrm{ml}$ ). Then, the Solvent was removed by heating the solution at $40^{\circ} \mathrm{C}$ for 6 hours in ambient pressure followed by applying vacuum for 24 hours. The dry lipid film was then hydrated through $1 \mathrm{ml} \mathrm{of} 15 \mathrm{mg} / \mathrm{ml} \mathrm{su}-$ crose aqueous solution to obtain multilamellar vesicles followed by lyophilization $\left(-52^{\circ} \mathrm{C}, 2.5 \mathrm{~Pa}\right)$ for 24 hours to achieve a preservable white powder. Unilamellar vesicles were also obtained through extrusion of multilamellar liposome suspension using a mini-extruder prior to lyophilization using a $100 \mathrm{~nm}$ polycarbonate membrane. The liposomes were reconstituted through addition of water to the obtained powder when required.

Particle size and poly dispersity measurements: In order to investigate the effect of lyophilization on vesicle formation, the mean particle size and size distribution of liposomes before and after lyophilization were determined using particle size analyzer (Brookhaven Instruments). All the measurements were performed at room temperature using water as the solvent.

Statistical Analysis: Two-tailed Student's t-test was used for the test of statistical significance using Microsoft Excel 2007 software (Microsoft, Redmond, WA). Calculating p-value, the level of the presumption against null hypothesis was determined for every experiment. Design-Expert software was used to prepare three-dimensional diagrams.

\section{Results}

The values of polydispersity of liposomes before and after lyophilization and extrusion processes are shown in Figure 1. It is seen that lyophilization has a dramatic effect on the poly dispersity index of particles in such a way that the values had twofold increase after lyophilization. Additionally, simultaneous effect of lyophilization and extrusion processes on the size of liposomes is presented in Figure 2. As it is obvious in this figure, size of liposomes decreased through extrusion. Despite using a $100 \mathrm{~nm}$ polycarbonate membrane, liposomes of about $200 \mathrm{~nm}$ were obtained through extrusion which might be a result of incorporation of a large amount of cholesterol in the formulation. When mulilamellar vesicles lyophilized size of them decreased slightly. This trend was reverse in the case of unilamellar vesicles and lyophilization increased the sizes. (Figure 3)

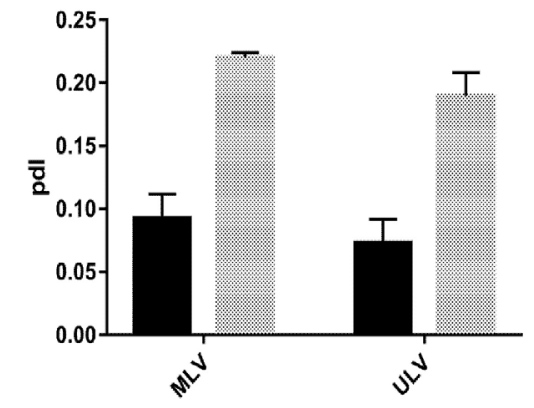

before lyophilization 2.: after lyophilization

\begin{tabular}{|l|l|l|l|}
\hline \multicolumn{2}{|l|}{ PdI of multilamellar vesicles } & \multicolumn{2}{l|}{ PdI of unilamellar vesicles } \\
\hline Before lyophilization & After lyophilization & Before lyophilization & After lyophilization \\
\hline $0.094 \pm 0.017$ & $0.220 \pm 0.004$ & $0.073 \pm 0.019$ & $0.190 \pm 0.018$ \\
\hline
\end{tabular}

Figure 1: Poly Dispersity Index (PdI) of liposomes before and after lyophilization and extrusion is presented in the figure above and the values are in the table below (MLV $=$ Multilamellar Vesicle, ULV $=$ Unilamellar Vesicle)
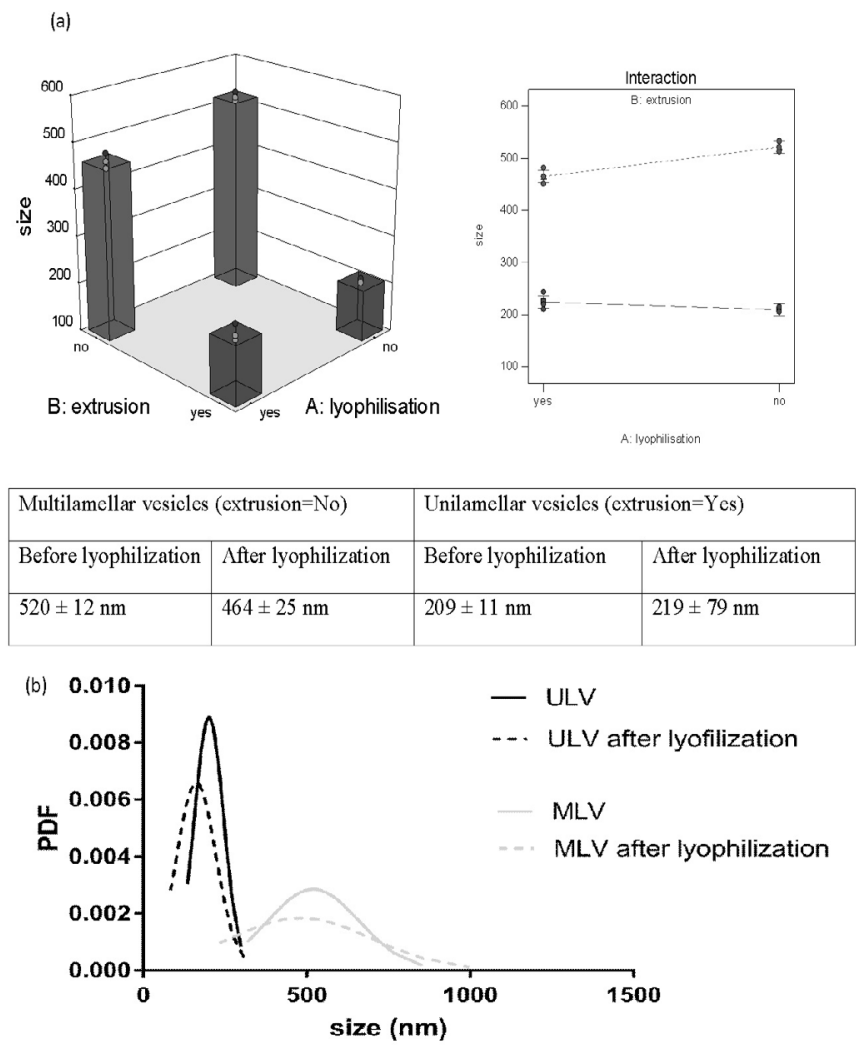

Error! Reference source not found. (a) figures are prepared by Design Expert Software showing mean particle size of liposomes before and after lyophilization and extrusion, (b) size diagram of four samples of unilamellar and multilamellar liposomes are compared. (PDF= Probability density function)

Figure 2: (a) Figures are prepared by Design Expert Software showing mean particle size of liposomes before and after lyophilization and extrusion, (b) Size diagram of four samples of unilamellar and multilamellar liposomes are compared. (PDF $=$ Probability density function) 


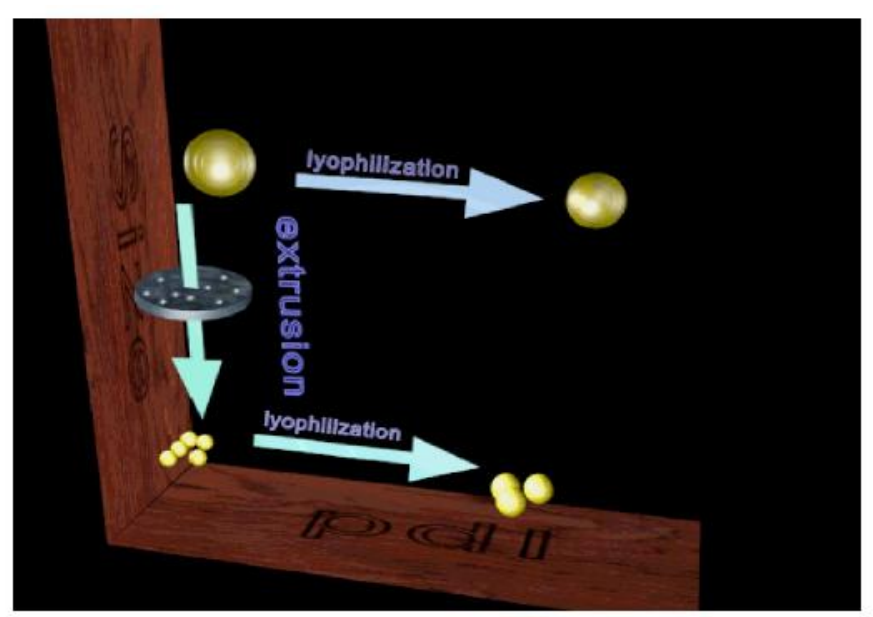

Figure 3: Schematic representation of effect of extrusion and lyophilisation on mean particle size and PdI of liposomes (figures is prepared by 3D MAX software).

\section{Discussion}

Cationic liposomes of DOTAP/DOPE/Cholesterol were prepared through a modified dry lipid film method. The amphiphilic nature of lipids causes self-assembly in aqueous media and multilamellar vesicles are produced via sonication. Extrusion of multilamellar vesicle suspension in the next step is expected to produce unilamellar vesicles ${ }^{[18]}$. Even though the dry lipid film hydration method is a simple and efficient procedure to produce liposomes, chemical and physical instability of vesicles in the aqueous media restricts the storage time of the formulation. Oxidative and hydrolytic degradation pathways might limit the shelf life of liposome dispersions. Furthermore, the average particle size and size distribution of liposome dispersions may change dramatically due to vesicle aggregation and fusion ${ }^{[19]}$. To address these problems, lyophilization was employed in which sucrose acted as the cryoprotectant in order to reduce the vesicle agglomeration and instability during the procedure.

It is well known that stabilization effect of cholesterol results in a more homogenous and stable liposome membrane. The presence of cholesterol in the membrane of DOTAP containing liposomes has been reported to cause a significant increase in size compared to cholesterol-free vesicles ${ }^{[20]}$. As could be seen in the Figure 2 the mean particle size had no significant change ( $p>0.1)$ while the PdI increased for both types of liposomes after lyophilization. Sucrose as the cryoprotectant in our formulation is expected to protect the vesicles and maintain the integrity of bilayers in the absence of water. This task is done via preventing fusion and rupture of bilayers by ice crystals. Sucrose may form an amorphous, glassy matrix inside and around the liposomes while interacted with lipid head groups. In addition, sucrose may reduce the stress on bilayers caused by drying ${ }^{[21]}$.

Indeed, the exact mechanism by which sugars like sucrose protect the liposome bilayer during lyophilization process is not determined yet. However, two hypotheses have been concentrated more; water replacement model and vitrification mod$\mathrm{e}^{[22]}$. In the first mechanism, the sugars are assumed to lessen interactions between the water and then replace the water while in the second one; formation of a sugar glass matrix is believed to protect lipid bilayers from damage by ice crystals. Both of the mechanisms have been proved to be required for lyophiliza- $\operatorname{tion}^{[23,24]}$

In fact, formulation and technological factors may affect the quality of protection during lyophilization. In our formulation, both positively charged lipid, DOTAP, and cholesterol could improve protection by improving stability. The $\mathrm{OH}$ moiety of cholesterol can interact with the $\mathrm{C}=\mathrm{O}$ and $\mathrm{P}=\mathrm{O}$ moieties of the lipids (i.e. DOTAP and DOPE) ${ }^{[25]}$; furthermore, cholesterol may weaken the interaction between the acyl chains and consequently prevent aggregation or improve the interaction between the lipids and cryoprotectant ${ }^{[26,27]}$. The cryoprotectant selection, the mass ratio of cryoprotectant/lipid and distribution of the cryoprotectant on the two sides of the bilayers are also of great importance. Disaccharides such as sucrose used in our formulation have been demonstrated to be effective in protecting membrane integrity ${ }^{[28]}$. Using our method of preparation, the sucrose is distributed on both sides of bilayers which results in better stability of liposomes compared to the presence of cryoprotectant on one side according to Crowe et al ${ }^{[29]}$.

Moreover, the particle size of the vesicles influences the outcome of the lyophilization procedure; for smaller vesicles because of fusion or aggregation processes, the particle size tends to increase while for the larger liposomes, fragmentations of vesicles might occur ${ }^{[30]}$. However, authors have frequently reported the increase of both mean particle size and $\mathrm{Pd} \mathrm{I}^{[31]}$ which might be a result of vesicle aggregation during the freezing step of lyophilization. Presumably, vesicles diffuse away from ice crystals toward the unfrozen fraction. Consequently, the particles are concentrated in the unfrozen fraction and aggregation take place ${ }^{[32]}$. In the case of our formulation, mean particle size remained almost unchanged $(\mathrm{p}>0.1)$ while the significant change of PdI was observed after lyophilization $(p<0.05)$. Nevertheless, one may notice mean particle size reduction of multilamellar vesicles through lyophilization despite it is insignificant as mentioned. Here it is worth mentioning that decrease in mean vesicle size through lyophilization is also possible. Vincourt et al. examined the effect of cryoprotectant (sucrose)/lipid ratio on the particle size of SPC/Cholesterol/DOTAP liposomal formulations. Interestingly, particle size declined significantly through lyophilization when a high ratio of sucrose/lipid (10:1) was employed $^{[33]}$. In another interesting study, Wieber et al. reported a decrease in the size of DOTAP/Cholesterol multilamellar vesicles after freeze-drying ${ }^{[20]}$. They assumed that the freezing process effect the liposome size ${ }^{[34]}$ as by freezing only the size was observed to decrease in a comparable manner.

It could be seen from the Figure 2 that the mean particle size of vesicles increases when DNA is added to the liposomes which are in agreement with the data reported by other authors ${ }^{[35,36]}$. Observing the dequenching of the fluorescent probe NBD-PE incorporated along with Rh-PE into the liposomes membrane, Radwan Almofti et al. found DNA-induced liposome-liposome responsible for the change in liposome size, upon addition of $\mathrm{DNA}^{[35]}$.

\section{Conclusion}

In this study, a lyophilized liposomal formulation of DOTAP/DOPE/Cholesterol was prepared through a modified lipid film hydration method. Extrusion through a membrane filter was employed to downsize the liposomes and produce unilamellar vesicles. Lyophilization found to change the PdI but not 
Polydispersity of Unilamellar and Multilamellar Liposomes the mean particle size of the vesicles significantly when sucrose used as the cryoprotectant.

\section{References}

1 Fonte, P., Soares, S., Sousa, F., et al. Stability Study Perspective of the Effect of Freeze-Drying Using Cryoprotectants on the Structure of Insulin Loaded into PLGA Nanoparticles. (2014) Biomacromolecules 15(10): 3753-3765.

2 Abdelwahed, W., Degobert, G., Stainmesse, S. et al. Freeze-drying of nanoparticles: Formulation, process and storage considerations. (2006) Adv Drug Deliv Rev 58(15): 1688-1713.

3 Choi, M.J., Briançon, S., Andrieu, J., et al. Effect of Freeze-Drying Process Conditions on the Stability of Nanoparticles. (2004) Drying Technology 22(1-2): 335-346.

4 Torchilin, V.P. Recent advances with liposomes as pharmaceutical carriers. (2005) Nat Rev Drug Discov 4(2): 145-160.

5 Eloy, J.O., Claro de Souza, M., Petrilli, R., et al. Liposomes as carriers of hydrophilic small molecule drugs: Strategies to enhance encapsulation and delivery. (2014) Colloids Surf B Biointerfaces 123: 345-363.

6 Stark, B., Pabst, G., Prassl, R. Long-term stability of sterically stabilized liposomes by freezing and freeze-drying: Effects of cryoprotectants on structure. (2010) Eur J Pharm Sci 41(4): 546-555.

7 El-Nesr, O.H., Yahiya, S.A., El-Gazayerly, O.N. Effect of formulation design and freeze-drying on properties of fluconazole multilamellar liposomes. (2010) Saudi Pharm J 18(4): 217-224.

8 Benjakul, R., Panyarachun, B., Sarisuta, N. Preparation of dry reconstituted liposomal powder by freeze-drying at room temperature. (2011) J Liposome Res 21(1): 28-37.

9 Emami, J., Rezazadeh, M., Varshosaz, J., et al. Formulation of LDL Targeted Nanostructured Lipid Carriers Loaded with Paclitaxel: A Detailed Study of Preparation, Freeze Drying Condition, and In Vitro Cytotoxicity. (2012) J Nanomaterials 2012: 10.

$10 \mathrm{Li}, \mathrm{C}$., Deng, Y. A novel method for the preparation of liposomes: Freeze drying of monophase solutions. (2004) J Pharm Sci 93(6): $1403-$ 1414.

11 Bangham, A.D., Standish, M.M., Watkins, J.C. Diffusion of univalent ions across the lamellae of swollen phospholipids. (1965) J Mol Bio 13(1): 238-252.

12 Shew, R.L., Deamer, D.W. A novel method for encapsulation of macromolecules in liposomes. (1985) Biochim Biophys Acta 816(1): 1-8.

13 Kremer, J.M., Esker, M.W., Pathmamanoharan, C., et al. Vesicles of variable diameter prepared by a modified injection method. (1977) Biochemistry 16(17): 3932-3935.

14 Szoka, F.Jr., Papahadjopoulos, D. Procedure for preparation of liposomes with large internal aqueous space and high capture by reverse-phase evaporation. (1978) Proc Natl Acad Sci U S A 75(9): 41944198.

15 Milsmann, M.H., Schwendener, R.A., Weder, H.G. The preparation of large single bilayer liposomes by a fast and controlled dialysis. (1978) Biochim Biophys Acta 512(1): 147-155.

16 Venkateswarlu, V., Manjunath, K. Preparation, characterization and in vitro release kinetics of clozapine solid lipid nanoparticles. (2004) J Control Release 95(3): 627-638.

17 Berger, N., Sachse, A., Bender, J., et al. Filter extrusion of liposomes using different devices: comparison of liposome size, encapsulation efficiency, and process characteristics. (2001) Int J pharm 223(2): 55-68. 18 Dhandapani, N.V., Thapa, A., Sandip, G., et al. Liposomes as novel drug delivery system: A comprehensive review. (2013) Int J Res Pharm Sci 4(2): 187-193.

19 Grit, M., Crommelin, D.J. Chemical stability of liposomes: implications for their physical stability. (1993) Chem Phys lipids 64(3): 3-18. 20 Wieber, A., Selzer, T., Kreuter, J. Physico-chemical characterisation of cationic DOTAP liposomes as drug delivery system for a hydrophilic decapeptide before and after freeze-drying. (2012) Eur J Pharm Biopharm 80(2): 358-367.

21 Duzgunes, N. Liposomes. (2003) Elsevier Science.

22 Chen, C., Han, D., Cai, C., et al. An overview of liposome lyophilization and its future potential. (2010) J Control Release 142(3): 299-311. 23 Crowe, L.M., Reid, D.S., Crowe, J.H. Is trehalose special for preserving dry biomaterials? (1996) Biophys J 71(4): 2087-2093.

24 Tsvetkova, N.M., Phillips, B.L., Crowe, L.M., et al. Effect of Sugars on Headgroup Mobility in Freeze-Dried Dipalmitoylphosphatidylcholine Bilayers: Solid-State 31 P NMR and FTIR Studies. (1998) Biophys J 75(6): 2947-2955.

25 Bach, D., Wachtel, E. Phospholipid/cholesterol model membranes: Formation of cholesterol crystallites. (2003) Biochim Biophys Acta 1610(2): 187-197.

26 Popova, A.V., Hincha, D.K. Effects of cholesterol on dry bilayers: interactions between phosphatidylcholine unsaturation and glycolipid or free sugar. (2007) Biophys J 93(4):1204-1214.

27 Ohtake, S., Schebor, C., de Pablo, J.J. Effects of trehalose on the phase behavior of DPPC-cholesterol unilamellar vesicles. (2006) Biochim Biophy Acta 1758(1): 65-73.

28 Madden, T.D., Bally, M.B., Hope, M.J., et al. Protection of large unilamellar vesicles by trehalose during dehydration: retention of vesicle contents. (1985) Biochim Biophys Acta 817(1): 67-74.

29 Crowe, J.H., Crowe, L.M. Factors affecting the stability of dry liposomes. (1988) Biochim Biophys Acta 939(2): 327-334.

30 Chen, C., Han, D., Zhang, Y., et al. The freeze-thawed and freezedried stability of cytarabine-encapsulated multivesicular liposomes. (2010) Inter J Pharm 387(2): 147-153.

31 Chaudhury, A., Das, S., Lee, R.F., et al. Lyophilization of cholesterol-free PEGylated liposomes and its impact on drug loading by passive equilibration. (2012) Inter J Pharm 430(2): 167-175.

32 Allison, S.D., Molina, M.C., Anchordoquy, T.J. Stabilization of lipid/DNA complexes during the freezing step of the lyophilization process: the particle isolation hypothesis. (2000) Biochim Biophys Acta 1468(2): 127-138.

33 Vincourt, V., Nguyen, L., Chaumeil, J.C., et al. Freeze-drying of ATP entrapped in cationic, low lipid liposomes. (2010) Cryobiology 60(3): 262-270.

34 Siow, L.F., Rades, T., Lim, M.H. Characterizing the freezing behavior of liposomes as a tool to understand the cryopreservation procedures. (2007) Cryobiology 55(3): 210-221.

35 Radwan Almofti, M., Harashima, H., Shinohara, Y., et al. Cationic liposome-mediated gene delivery: biophysical study and mechanism of internalization. (2003) Arch Biochem Biophys 410(2): 246-253.

36 Moghaddam, B., McNeil, S.E., Zheng, Q., et al. Exploring the Correlation Between Lipid Packaging in Lipoplexes and Their Transfection Efficacy. (2011) Pharmaceutics 3(4): 848-864.
Ommega Online Publishers

Journal Title: Journal of Nanotechnology and Materials Science Journal Short Name: J Nanotech Mater Sci

\section{Journal ISSN: 2377-1372}

E-mail: nanoscience@ommegaonline.com

Website: www.ommegaonline.org 